\title{
Supporting Information: Detection and Characterization of Single Particles by Electrochemical Impedance Spectroscopy
}

Brian Roehrich, ${ }^{[a]}$ Eric Z. Liu, ${ }^{[a]}$ Ravit Silverstein, ${ }^{[b]}$ and Lior Sepunaru*[a]

[a] Department of Chemistry and Biochemistry, University of California Santa Barbara. Santa Barbara, CA, USA.

[b] Materials Department, University of California Santa Barbara. Santa Barbara, CA, USA.

*Email: sepunaru@ucsb.edu

$\underline{\text { Table of Contents }}$

$\begin{array}{ll}\text { Page } & \text { Content } \\ \text { S2 } & \text { Experimental procedures } \\ \text { S4 } & \text { Perturbation waveform and limitations - Table S1 } \\ \text { S6 } & \text { Comparison between AC amplitudes - Figures S1, S2, Table S2 } \\ \text { S8 } & \text { Ferrocenemethanol CV - Figure S3 } \\ \text { S9 } & \text { Particle sizing by SEM - Figure S4 } \\ \text { S10 } & \text { Probability of overlapping events calculation } \\ \text { S11 } & \text { Electrode size measurements - Figure S5 } \\ \text { S12 } & \text { Table of typical EIS fit parameters - Table S3, Figure S6 } \\ \text { S13 } & \text { Effect of beads on all EIS parameters - Figure S7 } \\ \text { S14 } & \text { Step detection and quantification procedure - Figure S8 } \\ \text { S15 } & \text { Amperometric } \Delta I / I_{S S} \text { distribution - Figure S9 } \\ \text { S16 } & \text { Exchange current density calculation - Table S4 } \\ \text { S17 } & \text { Comparison between 2.76 and 4.86 } \mu m \text { electrodes - Figures S10, S11 } \\ \text { S19 } & \text { SEM micrograph of deformed particle - Figure S12 }\end{array}$




\section{Experimental procedures}

Materials: Solutions of ferrocenemethanol (AK Scientific, 98\%), $\mathrm{Ru}\left(\mathrm{NH}_{3}\right)_{6} \mathrm{Cl}_{3}$ (Acros Organics, 98\%), and potassium chloride (Sigma-Aldrich, $\geq 99.0 \%$ ) were prepared using MilliQ water $(\geq 18.2 \mathrm{M} \Omega \cdot \mathrm{cm}$ ). $1 \mu \mathrm{m}$ radius polystyrene beads were purchased from Invitrogen (batch number 1348481).

Electrochemical measurements: All measurements were performed using an EPC10 Double Patch Clamp Amplifier (HEKA Electronik, Lambrecht, Germany) in the three electrode configuration. A platinum wire was used as the counter electrode and a silver wire as a pseudoreference electrode. The working electrodes used were carbon disks with radii 2.76 and $4.86 \mu \mathrm{m}$ (purchased from $\mathrm{CH}$ Instruments and BASi, respectively), as measured electrochemically by $\mathrm{Ru}\left(\mathrm{NH}_{3}\right)_{6}^{3+}$ reduction. The electrochemical cell was placed inside well-grounded dual Faraday cages to reduce external electromagnetic noise. The EPC10 "Cfast" function was used to measure and correct for stray capacitances which would be detrimental to the impedance measurements. "C-fast" was measured with all three electrodes inside the electrochemical cell, but before the addition of electrolyte solution to the cell. This corrects for parasitic instrumental and cabling capacitances, defects in the working electrode, etc; but does not affect the capacitance associated with the electrode/electrolyte interface.

All measurements were performed using $2 \mathrm{mM} \mathrm{FcMeOH}$ and $0.5 \mathrm{mM} \mathrm{KCl}$. For measurements using beads, $10 \mu \mathrm{L}$ of stock solution (18.27 $p M)$ was added to $5 \mathrm{~mL}$ of FcMeOH solution to yield a final bead concentration of $36 \mathrm{fM}$. The beads were dispersed in the solution by sonicating. Before each $45 \mathrm{~s}$ impedance measurement, the working electrode was polished on $1 \mu \mathrm{m}, 0.3 \mu \mathrm{m}$, and $0.05 \mu \mathrm{m}$ alumina slurry (Buehler) sequentially for 1 minute each. A silver wire was chosen as the pseudoreference electrode because electrolyte leaks from conventional reference electrodes (i.e. saturated calomel, $\mathrm{Ag} / \mathrm{AgCl}$ in $\mathrm{KCl}$, etc. $)^{1}$ can significantly change the ionic strength of the weakly-supported $(0.5 \mathrm{mM} \mathrm{KCl})$ analyte solution. Changes in ionic strength could affect both the particle impact frequency, which is controlled by electromigration, as well as the charge-transfer kinetics.

FFT-EIS: The FFT-EIS waveform was created using a simple Python script that adds together multiple timedomain sine waves. Details of the nature of the waveform are given in the next section. The script output 
the waveform in ASCll format, which was imported into the potentiostat control software (PATCHMASTER) and used to create a custom stimulus file. This file set the AC potential of the working electrode during EIS measurements, the second channel of the potentiostat was used to add a constant DC bias to the AC waveform. The AC waveform was filtered at $500 \mathrm{kHz}$ before it was applied to the working electrode. In measurements, the voltage and current were sampled at $100 \mathrm{kHz}$ after the current was filtered using a $30 \mathrm{kHz}$ Bessel filter.

Later, the recorded time-domain voltage and current were split into predefined time intervals (100 ms). They were Fourier transformed using a custom Python script, and the impedance spectrum was calculated for each time segment. The $30 \mathrm{kHz}$ Bessel filter has a noticeable impact on the high-frequency impedance, so each impedance spectrum was corrected for the filter's transfer function by the following procedure: the impedance spectrum of a $10 \mathrm{M} \Omega$ resistor was measured and saved. $|\mathrm{Z}|$ and phase $(\phi)$ correction factors were determined at each frequency such that the corrected spectrum had $|\mathrm{Z}|=10 \mathrm{M} \Omega$ and $\phi=0$ at all frequencies. These correction factors were then applied to every measured impedance spectrum.

Finally, each impedance spectrum was fit to the equivalent circuit using MEISP software (Kumho Petrochemical Co. Ltd.) to extract time-resolved equivalent circuit parameters.

Cross-sectional SEM imaging: The contact area between the beads and the electrode was evaluated from SEM (Apreo C FEG from ThermoFisher). The electrode was first immersed in the typical FcMeOH solution, and $+100 \mathrm{mV}$ (vs Ag wire) was applied until a single particle impact event was observed in the current versus time trace. The electrode was then dried in air overnight before SEM imaging. Images were acquired using a low vacuum detector (LVD) operating in a low vacuum operation mode. The differential pressure between the specimen chamber and the gun column is controlled using water vapor which in this experiment was set to $50 \mathrm{~Pa}$. This type of imaging mode has enabled us to mitigate charging of the uncoated sample while maintaining good image resolution using an intermediate accelerating voltage of up to $10 \mathrm{kV}$. 


\section{Perturbation waveform and limitations}

Table S1. Frequencies (and phases) used to construct the multi-sine EIS waveform.

\begin{tabular}{|l|l|l|}
\hline Index & Frequency/ Hz & Phase/ degree \\
\hline $\mathbf{1}$ & 100 & 0 \\
\hline $\mathbf{2}$ & 110 & 143 \\
\hline $\mathbf{3}$ & 170 & 4 \\
\hline $\mathbf{4}$ & 250 & 136 \\
\hline $\mathbf{5}$ & 370 & 250 \\
\hline $\mathbf{6}$ & 520 & 129 \\
\hline $\mathbf{7}$ & 750 & 12 \\
\hline $\mathbf{8}$ & 1000 & 164 \\
\hline $\mathbf{9}$ & 1300 & -5 \\
\hline $\mathbf{1 0}$ & 2100 & 46 \\
\hline $\mathbf{1 1}$ & 3100 & 78 \\
\hline $\mathbf{1 2}$ & 4400 & -53 \\
\hline $\mathbf{1 3}$ & 6400 & 134 \\
\hline $\mathbf{1 4}$ & 9000 & 25 \\
\hline $\mathbf{1 5}$ & 11000 & 59 \\
\hline $\mathbf{1 6}$ & 13000 & 50 \\
\hline $\mathbf{1 7}$ & 17000 & 154 \\
\hline
\end{tabular}

We adopted our EIS waveform from that reported by Popkirov and Schindler, ${ }^{2}$ removing every other frequency in order to increase $\mathrm{S} / \mathrm{N}$ by concentrating the perturbation power into fewer frequencies. The time-domain waveform was calculated by Equation (1),

$$
v(t)=\sum_{j=1}^{17} a_{j} \sin \left(2 \pi f_{j} t+\phi_{j}\right)
$$

Where $a_{j}$ is the amplitude, $f_{j}$ the frequency (from Table S1), and $\phi_{j}$ the phase (from Table S1) of the $j^{\text {th }}$ sine wave. Typically, $a_{j}$ was the same for all frequencies (an exception is discussed below, for the $r_{e}=4.86 \mu m$ electrode). The summed waveform $v(t)$ was then normalized to the desired AC amplitude. For example, a $\pm 50 \mathrm{mV}$ AC amplitude means that the maximum of $v(t)$ is $50 \mathrm{mV}$ and the minimum -50 mV, giving a $100 \mathrm{mV}$ peak-to-peak.

The chosen frequency range was sufficient to resolve both the charge-transfer (high frequency) and diffusional features of $\mathrm{FcMeOH}$ oxidation on the carbon ultramicroelectrodes used in this study. Spectral acquisition with a lowest frequency of $100 \mathrm{~Hz}$ can in principle be accomplished in 
$10 \mathrm{~ms}$, but we noticed unacceptable noise in measurements in this configuration. Thus, we measured spectra every $100 \mathrm{~ms}$, such that each spectrum contains 10 periods of the lowest frequency $(100 \mathrm{~Hz})$ which significantly improves $\mathrm{S} / \mathrm{N}$. Time resolution on the order of $1 / f_{\min }$ may not be feasible due to these $\mathrm{S} / \mathrm{N}$ considerations. 
3. Comparison between $\mathrm{AC}$ amplitudes
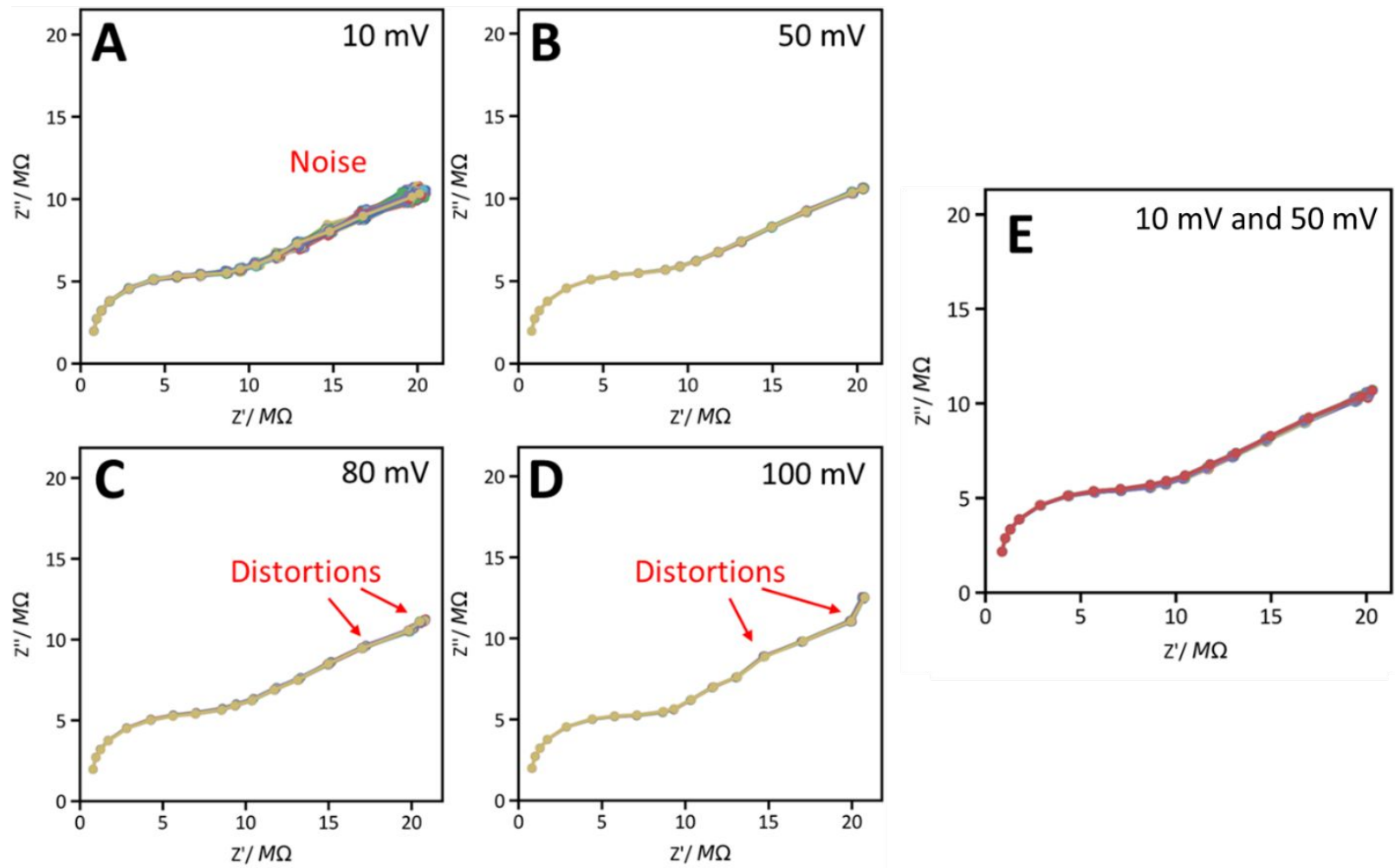

Figure S1. (A-D) Nyquist plots measured using (A) $10 \mathrm{mV}$, (B) $50 \mathrm{mV}$, (C) $80 \mathrm{mV}$, and (D) $100 \mathrm{mV}$ AC amplitude. Each measurement was recorded using an $r_{e}=2.76 \mu \mathrm{m}$ carbon UME in $2 \mathrm{mM}$ FcMeOH and $0.5 \mathrm{mM} \mathrm{KCl}$ in the absence of beads. The DC bias was the FcMeOH formal potential (+0.12 V vs Ag, equivalently $+0.194 \mathrm{~V}$ vs saturated calomel electrode). Each Nyquist plot contains 450 sequential spectra with $0.1 \mathrm{~s}$ measurement time, representing $45 \mathrm{~s}$ of continuous data. Noise is visible in the low-frequency dispersion in A), and distortions are visible in the low-frequency region of $C$ and $D$ due to either nonlinearities or current amplifier saturation. The $50 \mathrm{mV} A C$ amplitude in B allows for good noise performance without causing distortions in the Nyquist plot. (E) Overlayed Nyquist plots recorded with $10 \mathrm{mV}$ (yellow) and $50 \mathrm{mV}$ (red) AC perturbations. Each has 10 spectra with $1 \mathrm{~s}$ measurement time, which was chosen to limit the noise in the $10 \mathrm{mV}$ measurement (as is visible in A). The spectra overlap to the extent that the $10 \mathrm{mV}$ spectrum is entirely eclipsed, showing that the $50 \mathrm{mV}$ AC perturbation does not introduce significant nonlinearities compared to the $10 \mathrm{mV}$ perturbation. 

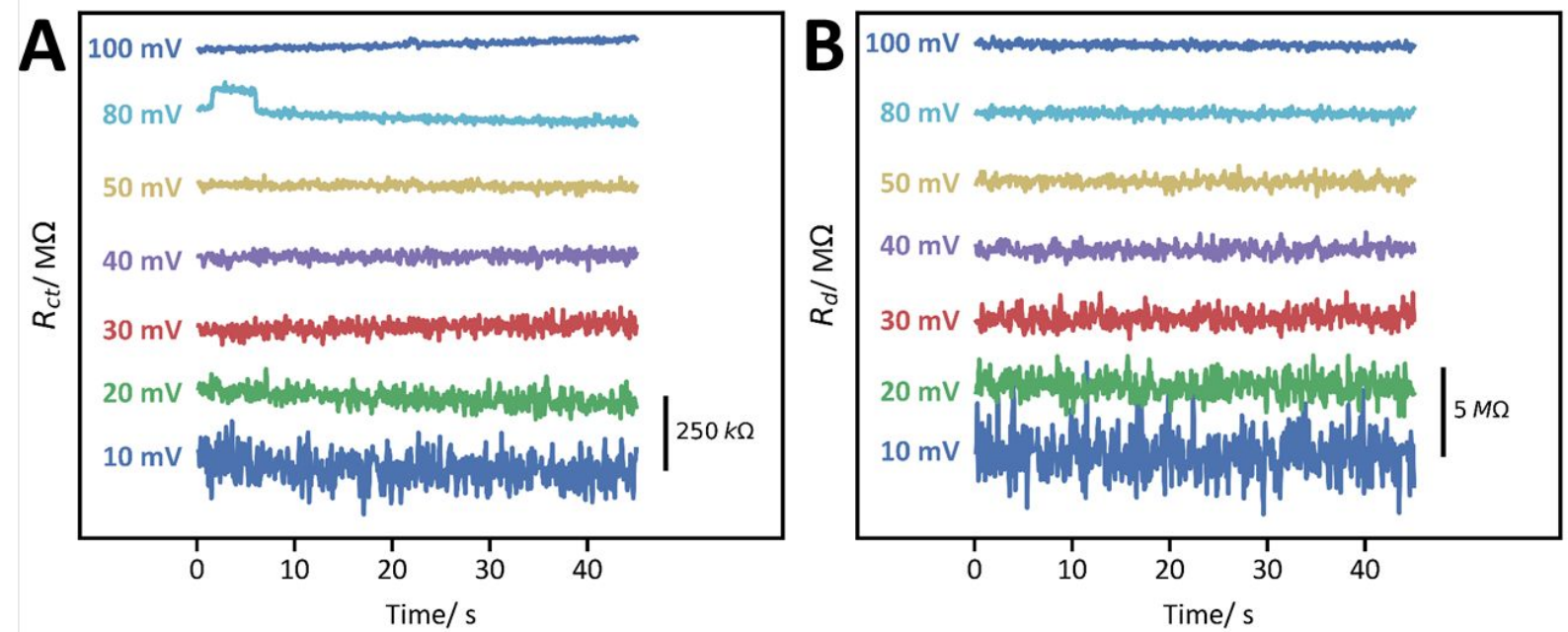

Figure S2. (A) $R_{c t}$ and (B) $R_{d}$ measured vs time with 7 different AC amplitudes (bottom to top: 10, $20,30,40,50,80$, and $100 \mathrm{mV}$ ) with $100 \mathrm{~ms}$ sampling. Each measurement is offset to allow visualization of the noise.

Table S2. Noise in $R_{c t}$ and $R_{d}$ as a function of AC amplitude.

\begin{tabular}{|c|c|c|}
\hline AC Amplitude/ $\mathbf{m V}$ & $\boldsymbol{R}_{\boldsymbol{c t}}$ Noise/ $\mathbf{M} \boldsymbol{\Omega}$ & $\boldsymbol{R}_{\boldsymbol{d}}$ Noise/ $\boldsymbol{M} \boldsymbol{\Omega}$ \\
\hline 10 & 0.054 & 1.29 \\
\hline 20 & 0.030 & 0.65 \\
\hline 30 & 0.022 & 0.49 \\
\hline 40 & 0.015 & 0.35 \\
\hline $\mathbf{5 0}$ & $\mathbf{0 . 0 1 1}$ & $\mathbf{0 . 2 9}$ \\
\hline 80 (distorted) & 0.010 & 0.22 \\
\hline 100 (distorted) & 0.013 & 0.20 \\
\hline
\end{tabular}


4. Ferrocenemethanol CV

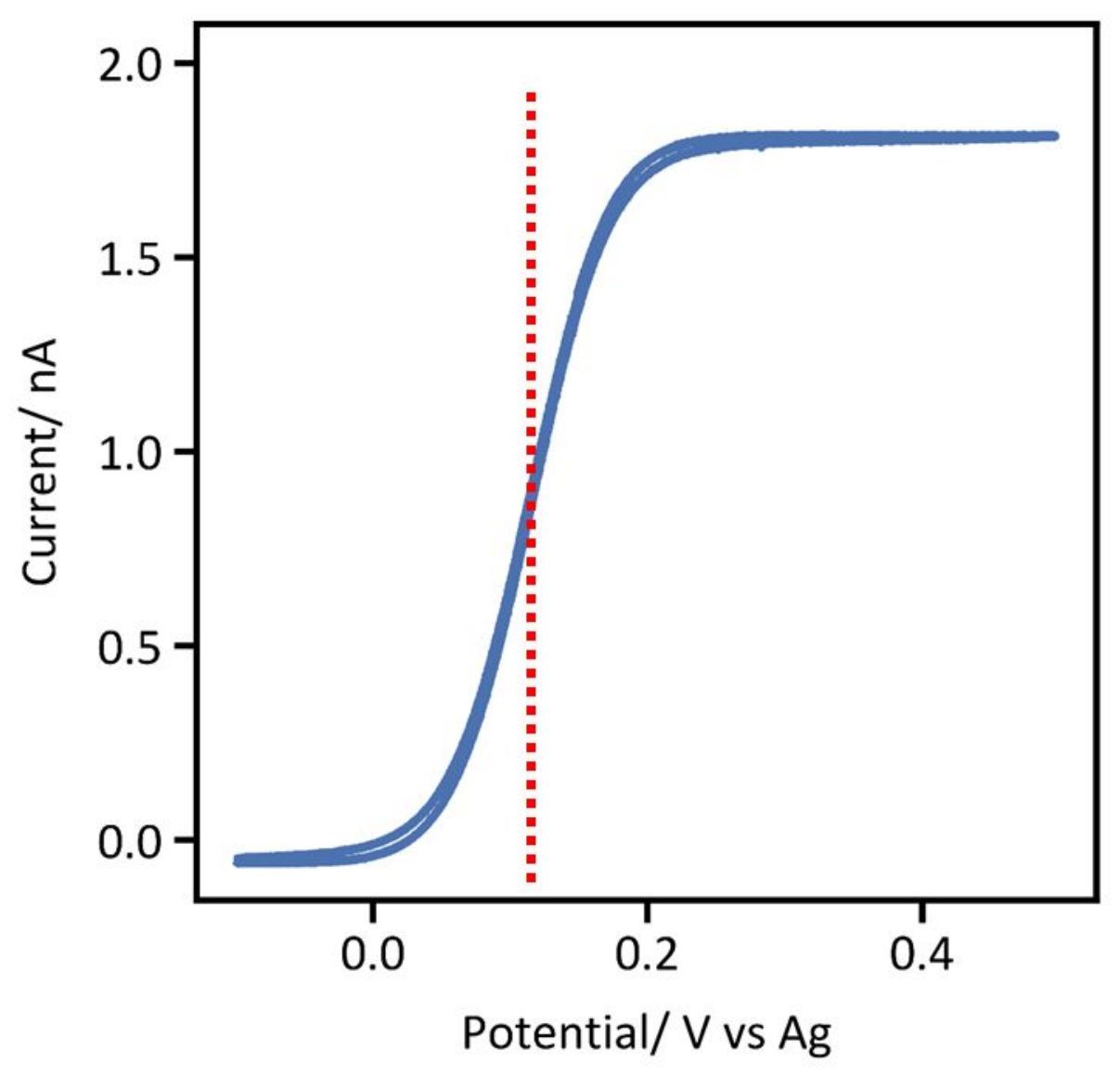

Figure S3. Cyclic voltammogram of $2 \mathrm{mM} \mathrm{FcMeOH}$ in $0.5 \mathrm{mM} \mathrm{KCl}$ recorded at $10 \mathrm{mV} / \mathrm{s}$ on the $r_{e}$ $=2.76 \mu \mathrm{m}$ carbon fiber ultramicroelectrode. The counter electrode was a Pt wire and the pseudoreference electrode was an Ag wire. The formal potential, which was applied as the DC bias in EIS measurements, is $+120 \mathrm{mV}$ vs $\mathrm{Ag}$ as indicated by the dashed red line. 
5. Bead SEM sizing
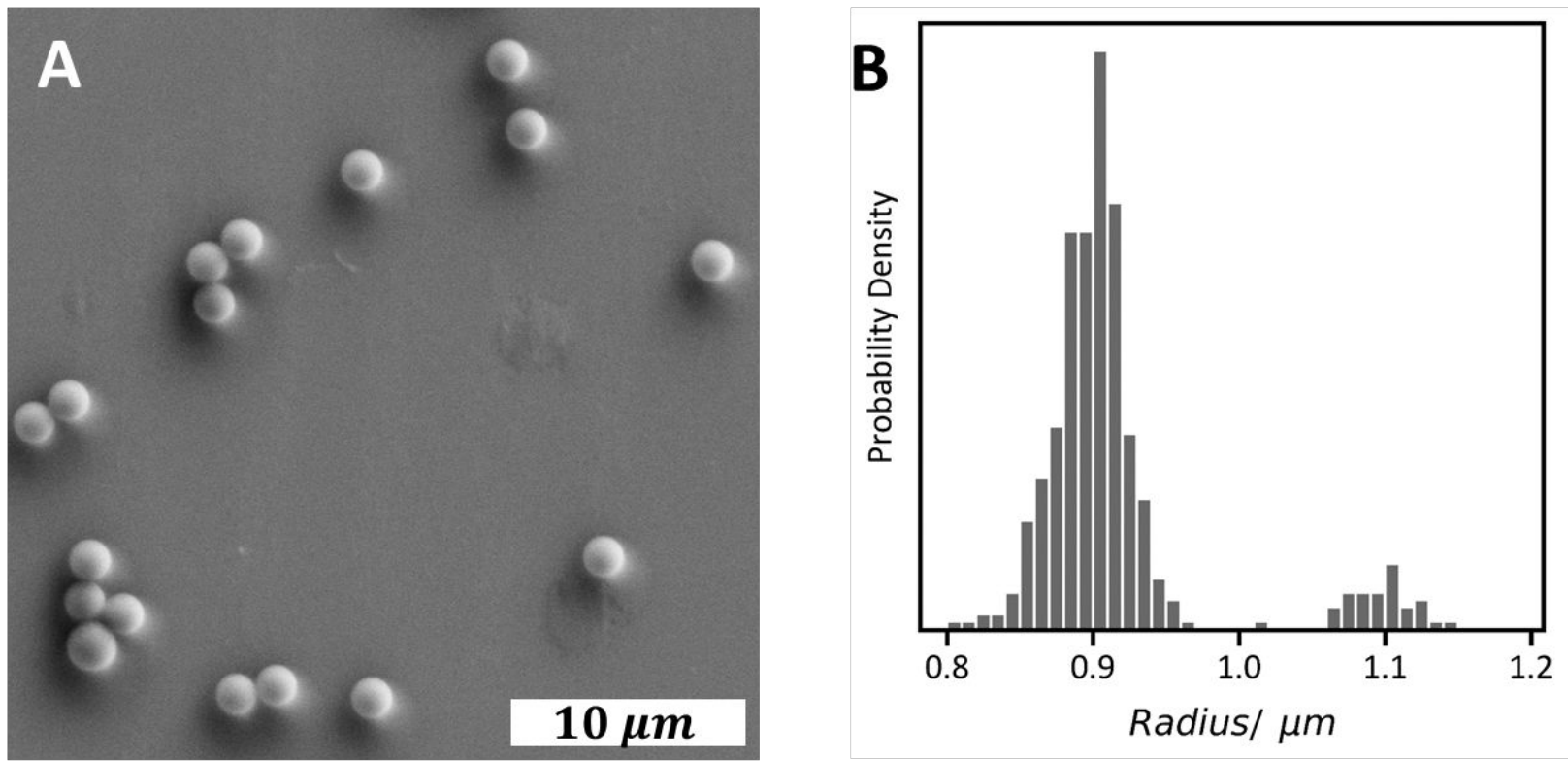

Figure S4. (A) Scanning electron micrograph of the $r_{b}=1 \mu \mathrm{m}$ beads adsorbed on a glass slide.

(B) Histogram of bead radii extracted from SEM micrographs using ImageJ $(\mathrm{N}=422)$. 
6. Probability of overlapping events calculation

We calculated the probability of two independent particles impacting within a 100 ms sampling interval. If this happened, data sampling would merge the two events into a single apparent impact. This can be modelled as a Poisson process because the events occur randomly, but with a known average frequency. ${ }^{3}$

$$
P(n)=\frac{e^{-\lambda t}(\lambda t)^{n}}{n !}
$$

Here, $\lambda=0.283 s^{-1}$ (average over $\mathrm{N}=337$ impact events), $t=0.1 s$, and $n=2$ impacts gives $P$ (2) $=4 \times 10^{-4}$. 


\section{Electrode size measurements}

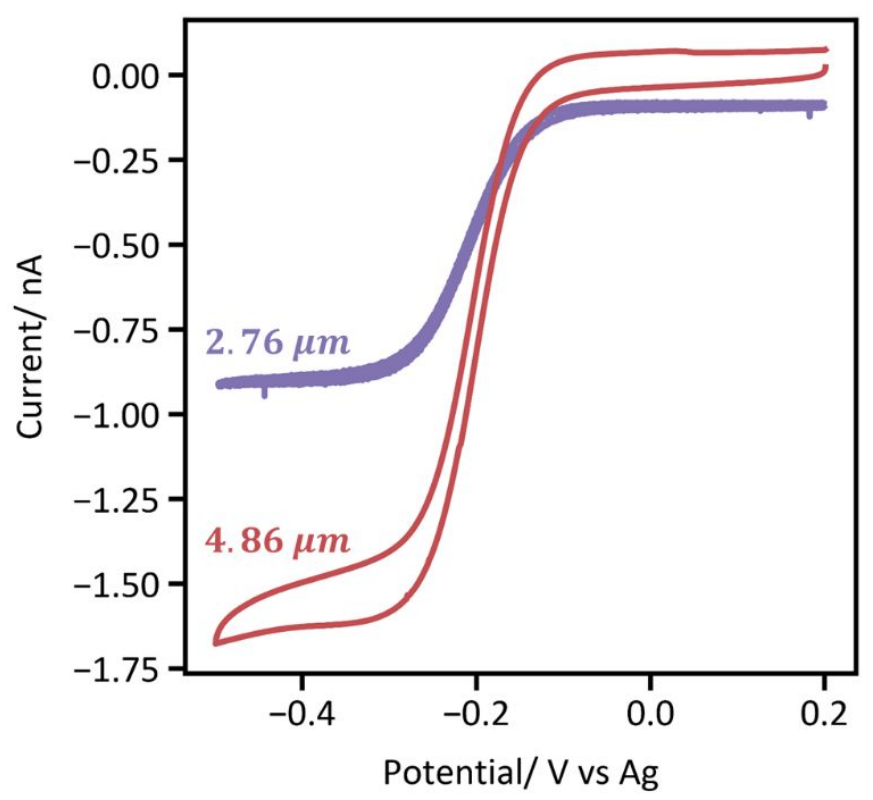

Figure S5. Cyclic voltammograms recorded using $r_{e}=2.76 \mu \mathrm{m}$ and $r_{e}=4.86 \mu \mathrm{m}$ carbon fiber ultramicroelectrodes in $1 \mathrm{mM} \mathrm{Ru}\left(\mathrm{NH}_{3}\right)_{6}{ }^{3+}$ and $100 \mathrm{mM} \mathrm{KCl}$ with a scan rate of $10 \mathrm{mV} / \mathrm{s}$. After baseline subtraction, the steady-state currents were determined to be 0.90 and $1.58 \mathrm{nA}$, respectively. Taking the diffusion coefficient of $\mathrm{Ru}\left(\mathrm{NH}_{3}\right)_{6}{ }^{3+}$ as $8.43 \times 10^{-10} \mathrm{~m}^{2} \mathrm{~s}^{-1,4}$ the electrode radii were calculated using Equation 3:5

$$
I=4 n F D C r
$$

Ruthenium (III) hexamine was used for electrode sizing because in the low electrolyte conditions used in the $\mathrm{FcMeOH}$ measurements, steady-state currents can deviate significantly due to selfinduced convection at the electrode surface. ${ }^{6}$ 
8. Table of typical EIS fit parameters

Table S3. Table of typical EIS fit parameters

\begin{tabular}{|c|c|c|c|c|c|}
\hline$R_{s} / M \Omega$ & $\boldsymbol{R}_{c t} / \boldsymbol{M} \boldsymbol{\Omega}$ & $R_{d} / M \Omega$ & $C_{d l} / p F$ & $Z_{d} / \Omega^{-1} s^{n}$ & $\alpha_{Z_{d}}$ \\
\hline 0.38 & 9.22 & 40.8 & 3.70 & 3.89 & 0.74 \\
\hline
\end{tabular}

Previous reports on microelectrode EIS have used a Warburg element to describe diffusion, i.e. $\alpha_{Z_{d}}=0.5$. We consistently observed $\alpha$ around 0.75 , possibly due to self-induced convection (caused by the low supporting electrolyte conditions used here) influencing the mass transport of the redox probe. ${ }^{6}$
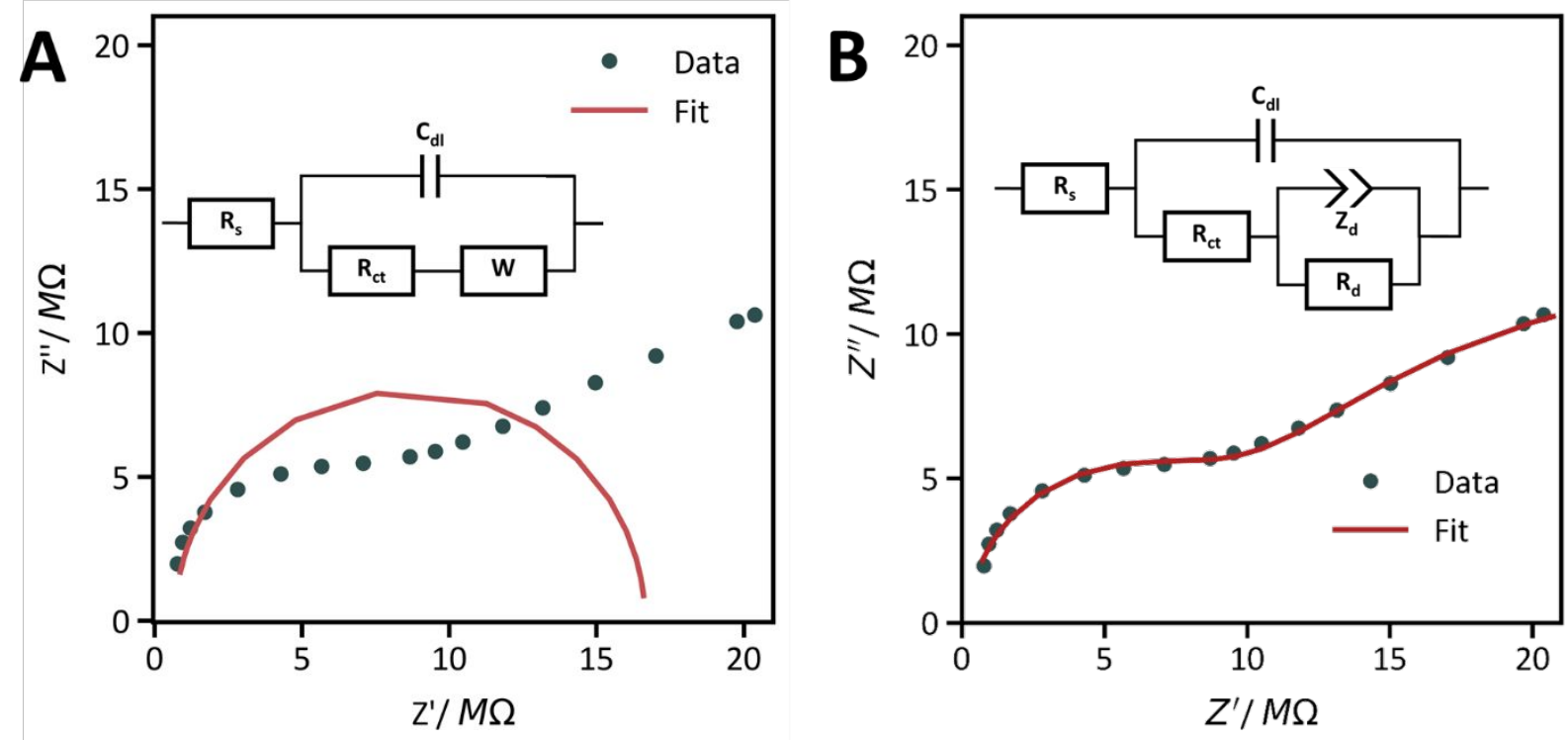

Figure S6. Comparison between fits from an inadequate equivalent circuit (Randles circuit, A) and the model used herein (B). The Randles circuit ( $A$, inset) uses a Warburg element (W) to model semi-infinite linear diffusion to a macroelectrode, rendering it unsuitable for the hemispherical diffusion to a microelectrode. 
9. Effect of beads on all EIS parameters
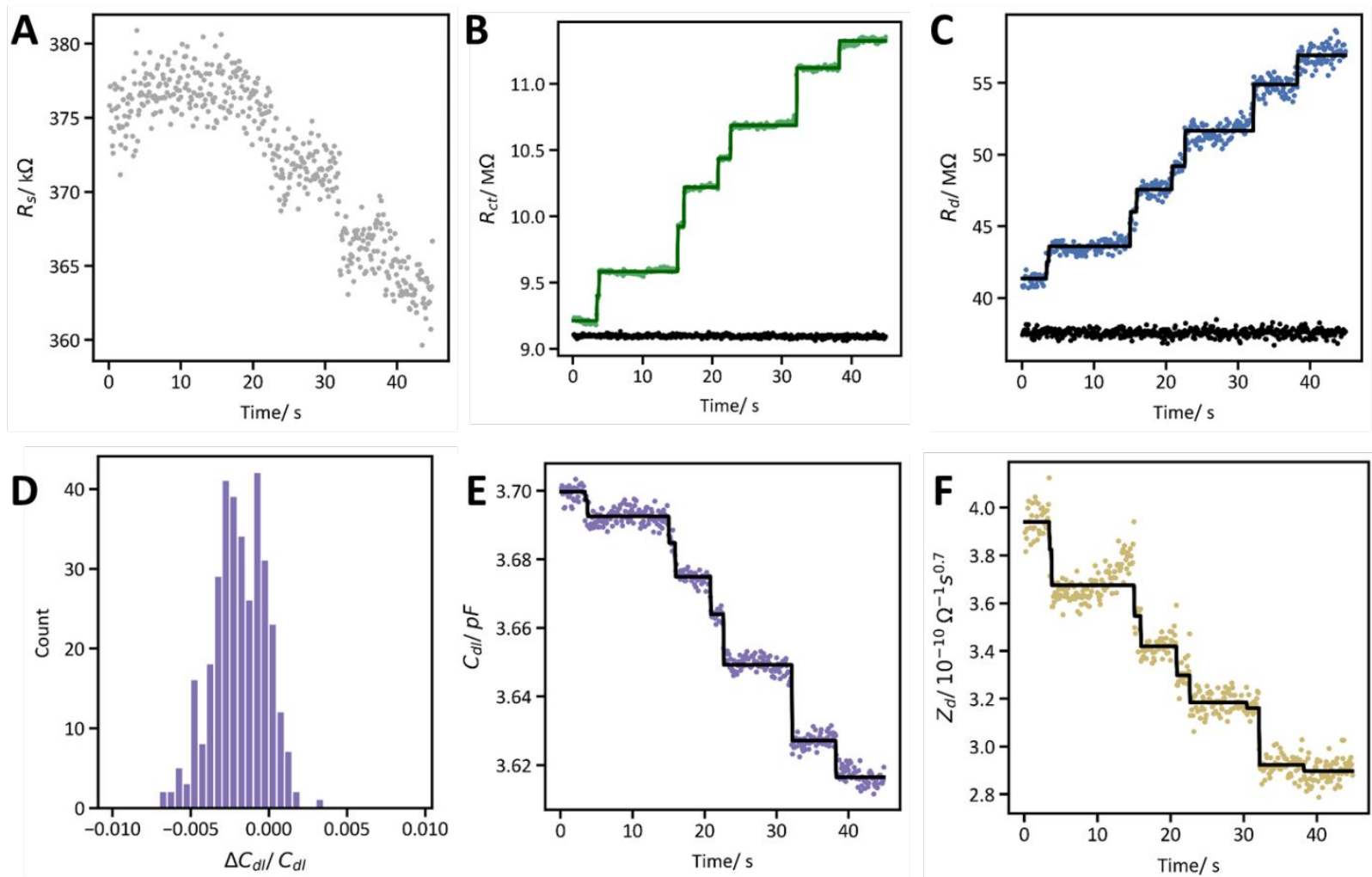

Figure S7. EIS equivalent circuit elements (A) $R_{S},(\mathrm{~B}) R_{c t},(\mathrm{C}), R_{d},(\mathrm{E}) C_{d l}$, and (F) $Z_{d}$ versus time. Data were recorded using an $r_{e}=2.76 \mu \mathrm{m}$ carbon UME in $2 \mathrm{mM} \mathrm{FcMeOH}$ and $0.5 \mathrm{mM} \mathrm{KCl}$ in the presence of $36 \mathrm{pM}$ polystyrene microbeads $\left(r_{b}=1 \mu \mathrm{m}\right)$. In addition to the $R_{c t}$ and $R_{d}$ data shown in the main text, $C_{d l}$ was analyzed using the same stair-stepping protocol. A histogram of $\Delta C_{d l} /$ $C_{d l}$ is shown in $\mathrm{D}\left(\mathrm{N}=337\right.$ steps). The majority of bead impacts caused a small decrease in $C_{d l}$, likely due to the adsorbed region of the bead inhibiting double layer formation. Decreases are also seen in $Z_{d}$ due to the hindered diffusion caused by the beads $(F)$, while no discrete events are observed in $R_{S}$. 


\section{Step detection and quantification procedure}

From the data shown in Figure $1 \mathrm{C}$ in the main text we determined that the steps were most prominent at $100 \mathrm{~Hz}$. We applied a simple stair-stepping algorithm to the $100 \mathrm{~Hz} \mid \mathrm{Z}$ | versus time data to determine time indices where bead impacts occurred (Figure S8 A, red triangles). For the $r_{e}=2.76 \mu \mathrm{m}$ electrode steps were picked with $\Delta|Z| /|Z|>1 \%$, while for the $r_{e}=4.86 \mu m$ electrode steps were picked with $\Delta|Z| /|Z|>0.5 \%$ due to the smaller signals. Then, the time indices were applied to the equivalent circuit parameters (for example, $R_{d}$ versus time as shown in Figure S8 B). The median value of the parameter between two sequential bead impacts was used to determine the step size. This approach let us extract usable data even from the relatively noisy $R_{d}$ data shown in Figure $\mathrm{S} 8 \mathrm{~B}$.
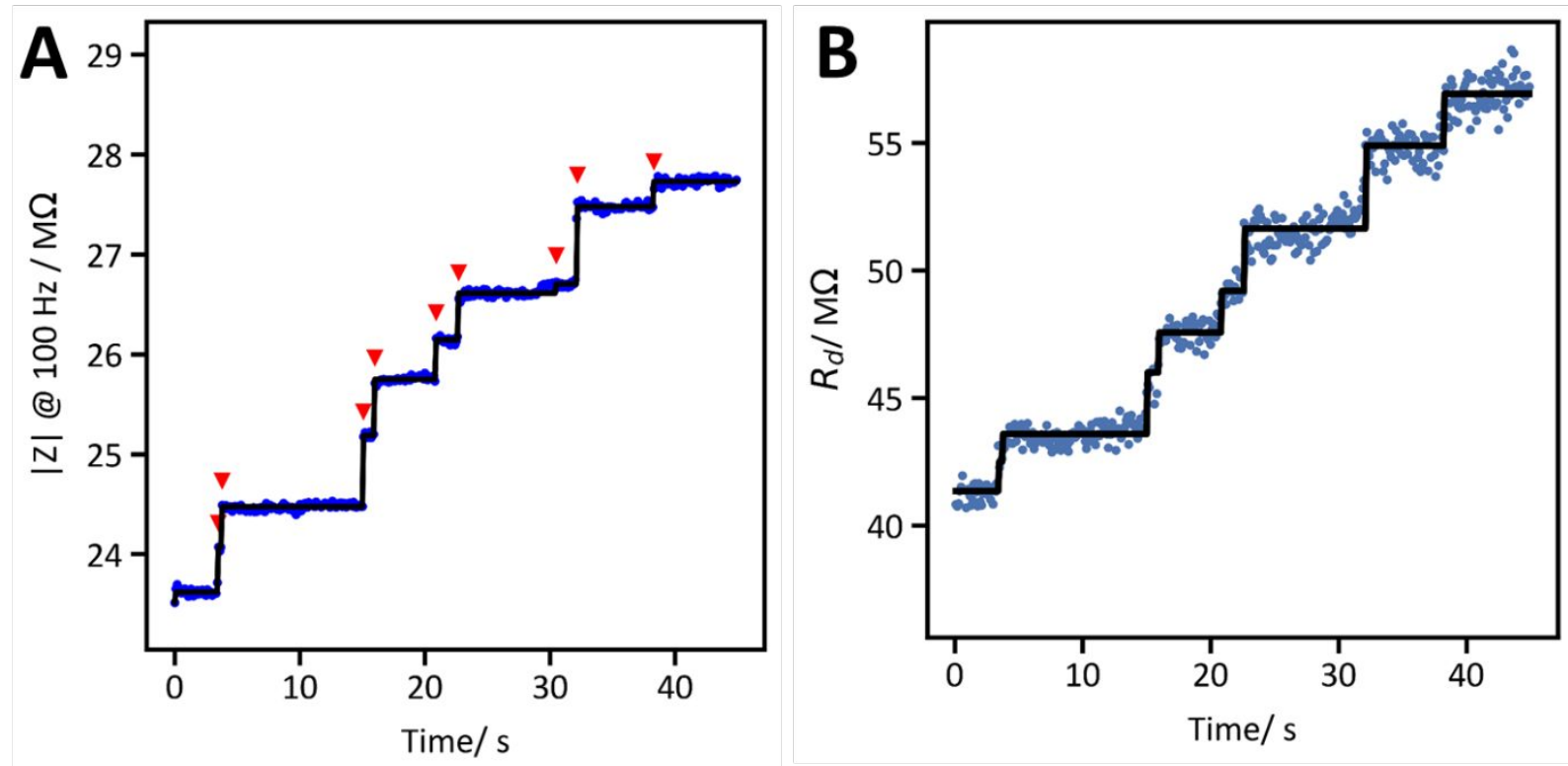

Figure S8. (A) Step detection algorithm applied to the $100 \mathrm{~Hz}|\mathrm{Z}|$ versus time. Steps (red triangles) are picked from the data (blue points). (B) $R_{d}$ versus time for the same dataset. The step times from A were used to fit a staircase response. The horizontal regions are the median value of $R_{d}$ between each step. 
11. Amperometric distribution
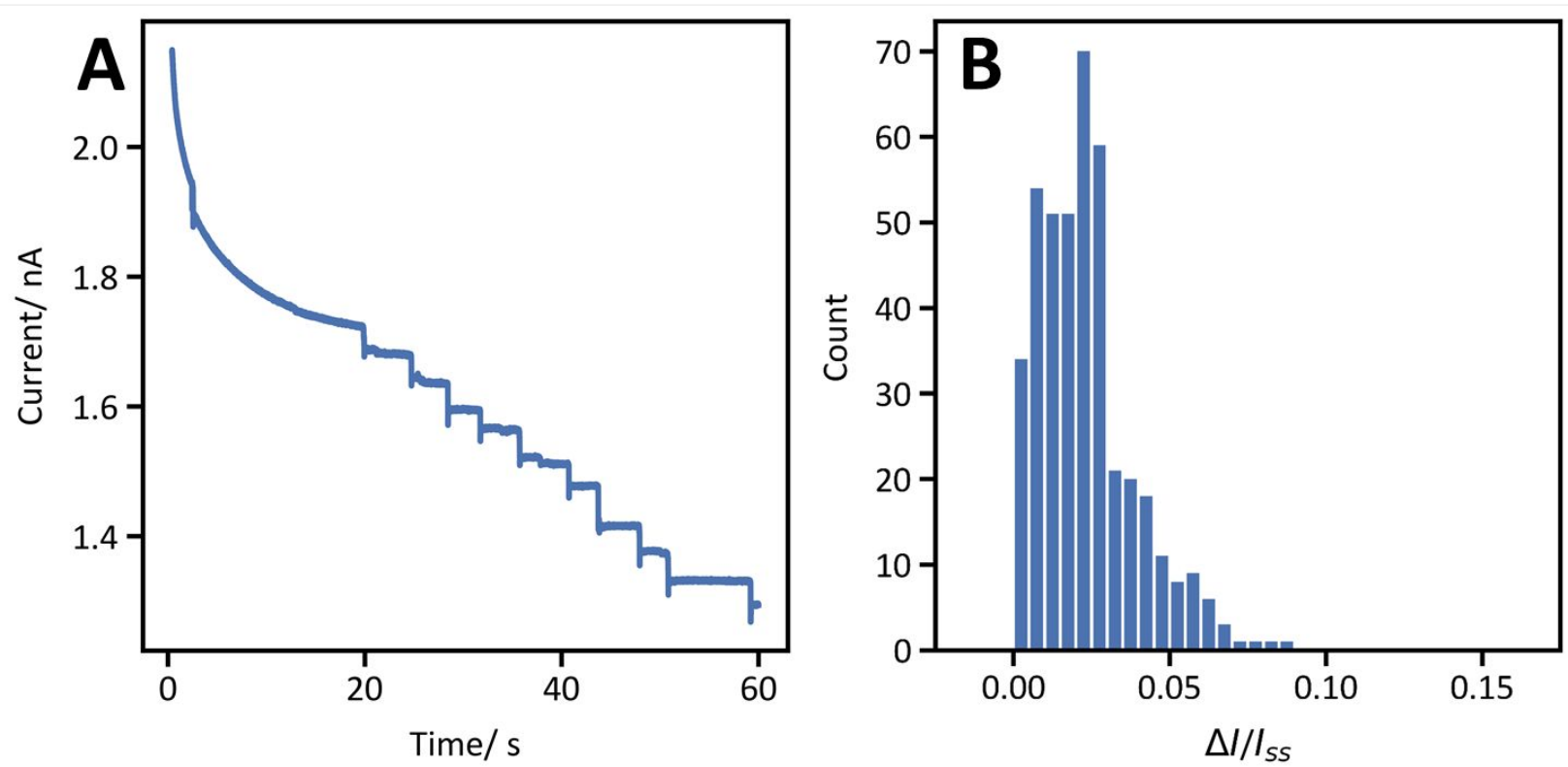

Figure S9. Amperometric detection of polystyrene microbeads. (A) Typical chronoamperomogram, recorded using a carbon ultramicroelectrode with $r_{e}=2.76 \mu m$ immersed in a solution of $2 \mathrm{mM} \mathrm{FCMeOH}, 0.5 \mathrm{mM} \mathrm{KCl}$ and $36 \mathrm{fM}$ polystyrene beads $\left(r_{b}=1 \mu \mathrm{m}\right)$. The working electrode was biased at $+400 \mathrm{mV}$ vs $\mathrm{Ag}$ wire, which is in the $\mathrm{FcMeOH}$ diffusionlimited regime. Each particle adsorption event discretely decreases current, manifesting in the steps visible in the figure. (B) Distribution of relative changes in current $\Delta I / I_{S S}$ corresponding to bead adsorption events measured using the amperometric technique $(N=419)$. 
12. Effective exchange current density calculation

The $R_{c t}$ parameter can be related to an effective exchange current density $j_{0, \text { eff }}$ as, ${ }^{7}$

$$
R_{c t}=\frac{R T}{n F A j_{0, e f f}}
$$

Where $R$ is the gas constant $\left(8.314 \mathrm{~J} \mathrm{~mol}^{-1} \mathrm{~K}^{-1}\right), T$ is temperature $(298 \mathrm{~K}), n$ is the number of electrons transferred (1), $F$ is Faraday's constant (96458 $\mathrm{C} \mathrm{mol}^{-1}$ ), and $A$ is the electrode surface area. $j_{0, e f f}$ is not the true exchange current density $j_{0}$ due to non-equilibrium conditions in the bulk solution (the initial conditions have no $\mathrm{FcMeOH}^{+}$in the bulk). However, it is constant over the measurement duration as seen by the control experiment in Figure $2 \mathrm{~B}$ in the main text and allows relation of $R_{c t}$ to the electrode area.

Table S4. $j_{0, e f f}$ values calculated from Equation (4).

\begin{tabular}{|c|c|c|c|}
\hline Electrode radius/ $\boldsymbol{\mu m}$ & Area/ $\boldsymbol{\mu m}^{\mathbf{2}}$ & $\boldsymbol{R}_{\boldsymbol{c t}} / \boldsymbol{M} \boldsymbol{\Omega}$ & $\boldsymbol{j}_{\mathbf{0}, \boldsymbol{e f f}} /{\mathbf{m A ~} \mathbf{c m}^{-\mathbf{2}}}$ \\
\hline 2.76 & 23.9 & 9.22 & 11.7 \\
\hline 4.86 & 55.7 & 6.37 & 7.2 \\
\hline
\end{tabular}

Equation (4) was used to calculate the electrode area shown in Figure 3B in the main text. 


\section{Comparison between 2.76 and $4.86 \mu m$ electrodes}

We also used single entity EIS to measure particle impacts on an $r_{e}=4.86 \mu \mathrm{m}$ electrode. To avoid saturating our current amplifier, we optimized the voltage waveform such that $a_{j} \propto|Z|_{j}$ as previously described by Popkirov and Schindler. ${ }^{8}$ This allowed a high-amplitude perturbation at low frequency (high impedance) to maximize $\mathrm{S} / \mathrm{N}$ while avoiding saturating the amplifier by passing a large current at high frequencies (low impedance). The waveform used is shown in Figure S10.

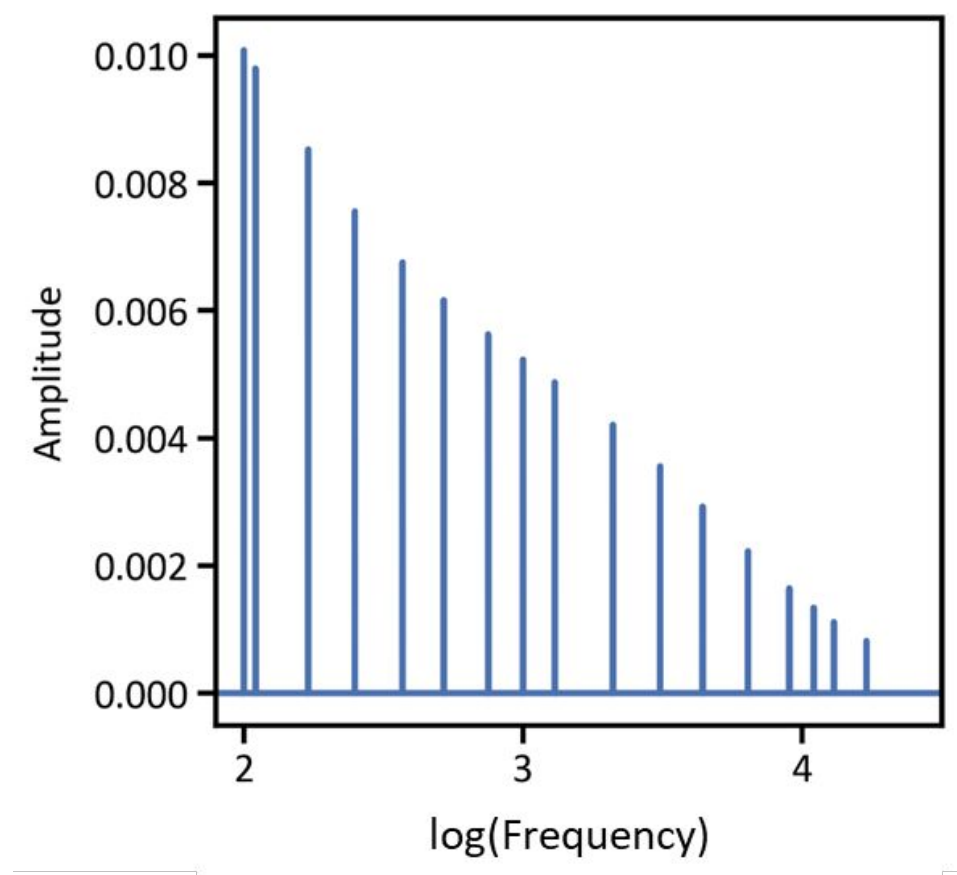

Figure S10. Frequency domain representation of the "optimized" waveform used with the $r_{e}$ $=4.86 \mu \mathrm{m}$. In the time domain, the summed voltage was still normalized to have a maximum of $50 \mathrm{mV}$. 

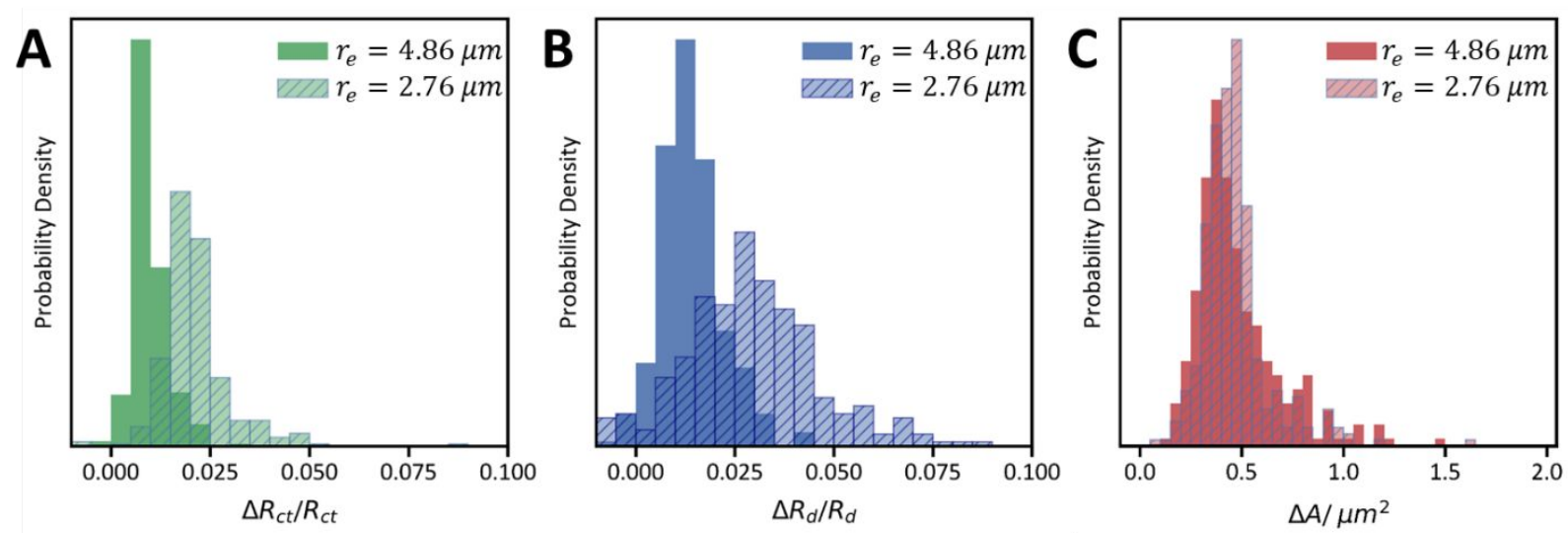

Figure S11. Comparisons in distributions of (A) $\Delta R_{c t} / R_{c t}$, (B) $\Delta R_{d} / R_{d}$, and (C) $\Delta A$ obtained on the $r_{e}=2.76 \mu \mathrm{m}$ electrode (dashed, $\mathrm{N}=337$ ) and those obtained on the $r_{e}=4.86 \mu \mathrm{m}$ electrode (solid, $\mathrm{N}=294$ ). The histograms shown in $\mathrm{C}$ are reproduced from Figure $3 \mathrm{~B}$ in the main text for comparison. Data were recorded in $2 \mathrm{mM} \mathrm{FcMeOH}$ and $0.5 \mathrm{mM} \mathrm{KCl}$ in the presence of $36 \mathrm{pM}$ polystyrene microbeads $\left(r_{b}=1 \mu \mathrm{m}\right)$. Despite shifts in $\Delta R_{c t} / R_{c t}$ and $\Delta R_{d} / R_{d}$ between the two electrode sizes, the distributions in $\Delta A$ have significant overlap below $0.5 \mu \mathrm{m}^{2}$. Relative step sizes in $A$ and $B$ are smaller on the large $(4.86 \mu \mathrm{m})$ electrode because each bead has a smaller relative effect on the electrode compared to the small $(2.76 \mu \mathrm{m})$ electrode. 
14. SEM micrograph of deformed particle

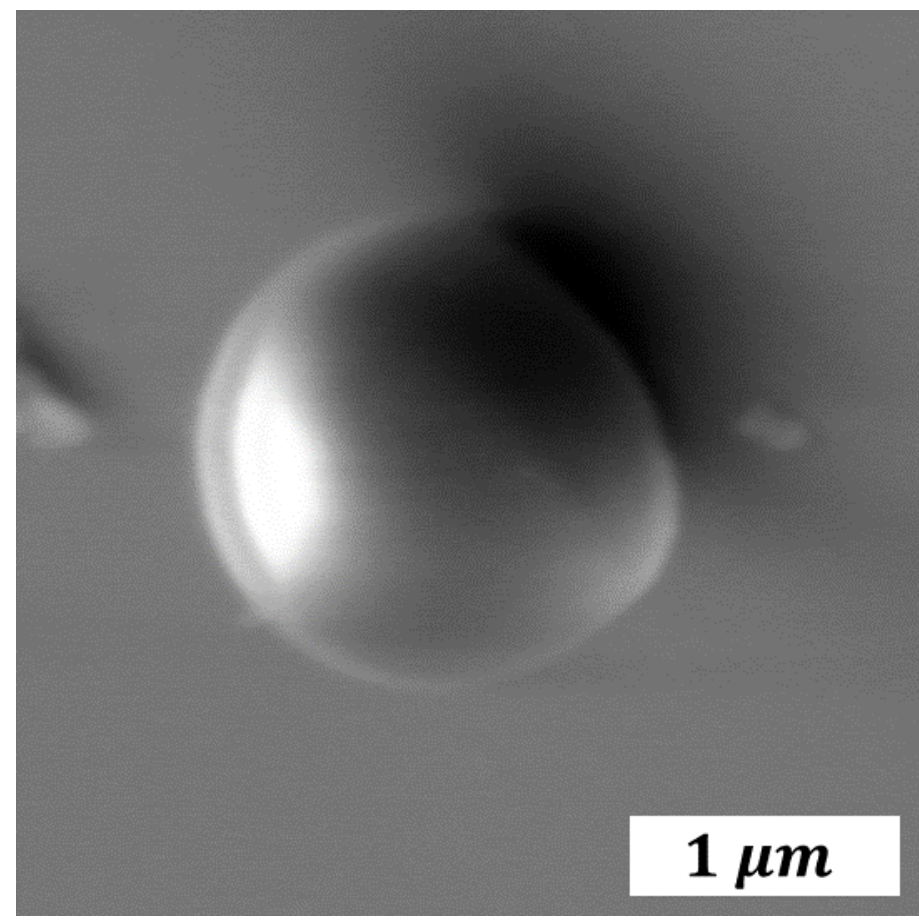

Figure S14. SEM micrograph of a polystyrene particle on the carbon fiber ultramicroelectrode surface. The particle is visibly deformed from the typical spherical geometry, which affects its contact area with the electrode. 


\section{References}

(1) Yakushenko, A.; Mayer, D.; Buitenhuis, J.; Offenhäusser, A.; Wolfrum, B. Electrochemical Artifacts Originating from Nanoparticle Contamination by $\mathrm{Ag} / \mathrm{AgCl}$ Quasi-Reference Electrodes. Lab Chip 2014, 14 (3), 602-607.

(2) Popkirov, G. S.; Schindler, R. N. A New Impedance Spectrometer for the Investigation of Electrochemical Systems. Rev. Sci. Instrum. 1992, 63 (11), 5366-5372.

(3) McElreath, R. Statistical Rethinking: A Bayesian Course with Examples in R and Stan; Chapman and Hall/CRC, 2015.

(4) Wang, Y.; Limon-Petersen, J. G.; Compton, R. G. Measurement of the Diffusion Coefficients of $\left[\mathrm{Ru}(\mathrm{NH} 3)_{6} 6\right] 3+$ and $\left[\mathrm{Ru}(\mathrm{NH} 3)_{6} 62+\right.$ in Aqueous Solution Using Microelectrode Double Potential Step Chronoamperometry. J. Electroanal. Chem. 2011, $652(1-2), 13-17$.

(5) Compton, R. G.; Banks, C. E. Understanding Voltammetry, 3rd ed.; World Scientific, 2018.

(6) Moazzenzade, T.; Yang, X.; Walterbos, L.; Huskens, J.; Renault, C.; Lemay, S. G. SelfInduced Convection at Microelectrodes via Electroosmosis and Its Influence on Impact Electrochemistry. J. Am. Chem. Soc. 2020.

(7) Bard, A. J.; Faulkner, L. R. Electrochemical Methods: Fundamentals and Applications, 2nd ed.; John Wiley \& Sons, 2001.

(8) Popkirov, G. S.; Schindler, R. N. Optimization of the Perturbation Signal for Electrochemical Impedance Spectroscopy in the Time Domain. Rev. Sci. Instrum. 1993, 64 (11), 3111-3115. 